\title{
Intramedullary thoracic spinal metastasis from small-cell lung cancer
}

\author{
S. Katsenos ${ }^{1}$, M. Nikolopoulou 2
}

ABSTRACT: Intramedullary thoracic spinal metastasis from small-cell lung cancer. S. Katsenos, M. Nikolopoulou.

Lung cancer with intramedullary spinal cord metastasis (ISCM) is a rare event exhibiting dismal prognosis. In the present paper, we describe a 74-year-old male who developed bilateral leg weakness with associated backache and non-productive cough. Chest imaging evaluation demonstrated pronounced bilateral mediastinal lymphadenopathy and a nodular opacity in the right lower lobe. The patient was diagnosed with small cell lung can- cer through bronchoscopic procedures. Magnetic resonance imaging of the spinal cord with contrast-enhancement revealed an intramedullary lesion consistent with metastasis at the T5-T6 level. Despite chemotherapy and thoracic spine radiotherapy, he eventually succumbed to the disease 3 months after diagnosis. A brief overview of the current literature is also provided laying emphasis on the therapeutic strategies of this unusual extrathoracic metastatic disease.

Monaldi Arch Chest Dis 2013; 79: 3-4, 140-142.

Keywords: Lung cancer, Intramedullary Spinal cord metastasis.

1 Department of Pneumonology, Arny General Hospital of Athens, Athens;

2 First Department of Pneumonology, General Hospital of Chest Diseases "Sotiria", Athens, Greece.

Correspondence: Stamatis Katsenos, MD, PhD, Department of Pneumonology, Army General Hospital of Athens, 158 Mesogion\&Katehaki Avenue, 11525 Athens, Greece; e-mail: skatsenos@yahoo.gr

Intramedullary spinal cord metastasis (ISCM) is a well-known but rare complication of systemic malignant tumors [1]. Its presence indicates a widespread systemic disease which progresses rapidly. Herein, we report a well-documented case of a small cell lung cancer with solitary thoracic spinal cord metastasis and include a short review of the recent literature regarding the clinical and therapeutic aspects of this unusual entity.

\section{Case Report}

A 74-year-old male, ex-smoker (40 pack/ years-smoking cessation 25 years ago), wasreferred to our department due to a weakness in his lower limbs accompanied by backache and nonproductive cough, gradually progressive over the last three months. His past medical history included total laryngectomy with permanent tracheostomy for squamous cell laryngeal cancer which he had undergone in 1982.

Chest physical examination revealed diminished breath sounds in the right middle and lower lung fields. Furthermore, proximal paresis of the legs was noted and muscle strength graded $2-3 / 5$ based on Medical Research Council (MRC) scale. Sensory function testing and especially pain sensation was slightly impaired below the L2 level in both legs.

Admission chest radiograph and contrast-enhanced computed tomography showed a right perihilar tumor mass accompanied by a nodular opacity in the right lower lobe (figure 1). Transbronchial needle aspiration from enlarged mediastinal lymph nodes as well as transbronchial biopsies using fluoroscopy all resulted positive for small cell lung cancer. Additional work-up using abdominal and brain $\mathrm{CT}$ and bone scan did not detect any extrathoracic metastases. However, gadolinium-based contrast magnetic resonance imaging of the spine demonstrated a spindle-like enhancing intramedullary lesion at the T5-T6 levels measuring $2.1 \mathrm{~cm}$ in diameter with surrounding edema (figure 2). These findings were compatible with metastatic disease.

The patient was recommended surgical resection of the intramedullary lesion because of its solitary location and his relatively good general condition (ECOG performance status: 2). Nevertheless, he refused any surgical intervention and consequently received palliative thoracic spine radiotherapy (30 Gy in 10 fractions) as well as systemic corticosteroids. After radiotherapy, he reported partial improvement in motor function of the lower extremities and back pain relief. Moreover, he was given combination chemotherapy regimen including cisplatin and etoposide. After just two cycles, he developed acute respiratory failure and CT imaging showed findings compatible with lymphangitis carcinomatosa thus reflecting a rapidly growing tumor burden. He eventually succumbed to the disease approximately three months after his initial diagnosis.

\section{Discussion}

Intramedullary spinal cord metastasis is a rare and tantalizing complication of malignancy. It has 


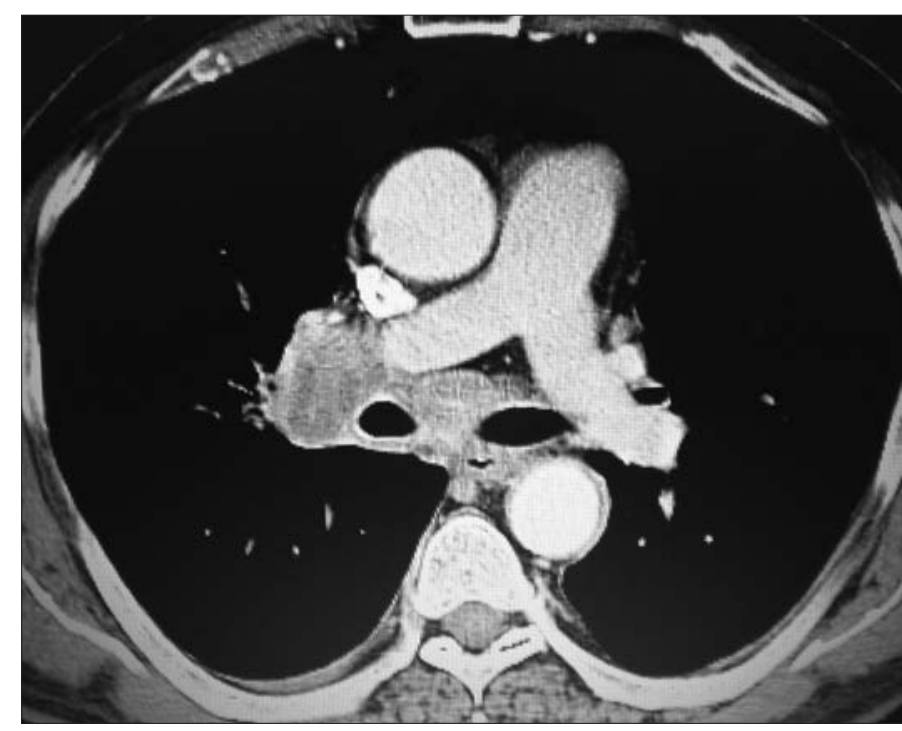

Fig. 1. - Contrast-enhanced chest computed tomography showing a right perihilar tumor mass accompanied by a nodular opacity in the right lower lobe.

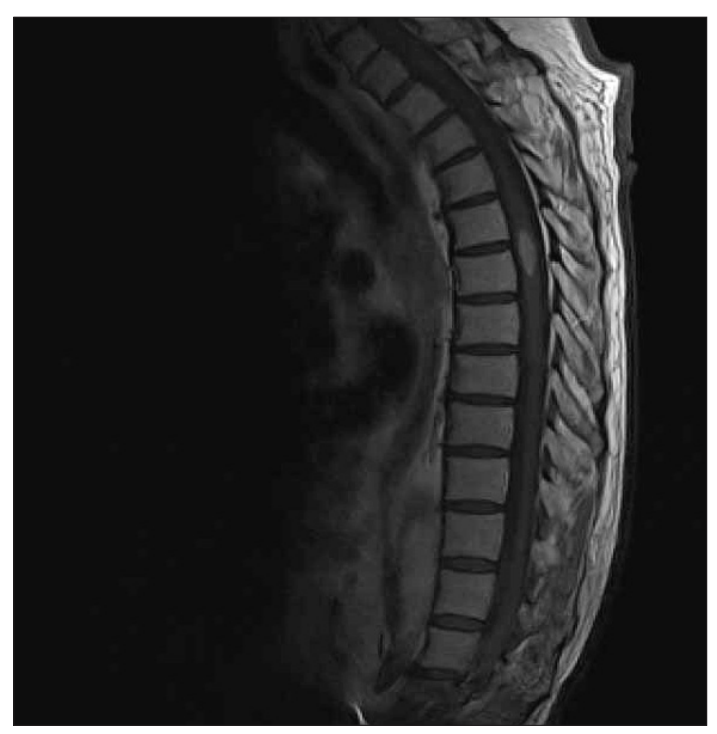

Fig. 2. - Contrast sagittal T1-weighted magnetic resonance imaging (MRI) of the thoracic spine showing a spindle-like enhancing intramedullary lesion at the T5-T6 levels. been reported in $0.9-2.1 \%$ of all neoplasm autopsies and usually coexists with brain or leptomeningeal metastases in cancer patients $[1,2]$. Additionally, ISCMs commonly present as solitary lesions. Multiple metastases are even rarer, with two lesions found in only $10 \%$ of patients [3]. Lung and breast carcinomas comprise the most common primary tumor sites in ISCM $[2,4]$.

ISCM usually occurs in patients with advanced stage diseases, thus suggesting rapidly progressing systemic malignancy. Occasionally, ISCM may occur as initial presentation of malignancy $[1,5]$. At diagnosis, the majority of patients with ISCM have a known primary cancer often associated with cerebral and other systemic metastases. The duration of symptoms before diagnosis ranges from 2 days to 8 months (median, 7 days) [4]. Common presenting symptoms are pain, motor weakness, sensory loss, and incontinence. Another manifestation is Brown-Sequard syndrome or complete spinal cord transaction which is observed in almost half of patients with ISCM [1].

With the advent of MRI diagnosis of ISCM has been greatly simplified. Moreover, contrastenhanced MRI may easily depict intramedullary spinal metastatic lesions with their associated edema, thus delineating the extent of lesions as well as regions of spinal cord compression [1]. Prior to the era of MRI, only 5\% of ISCMs were detected antemortem. Nowadays, positron emission tomography scanning combined with computed tomography (PET/CT) is gaining ground in the assessment of secondary malignant involvement of the spinal column resulting in increased sensitivity $(98 \%)$ [6].

Optimal management of ISCM is cumbersome due to its rarity, the wide variety of clinical aspects and a lack of controlled studies comparing the different therapeutic modalities involved. Currently, the surgical approach is less invasive by virtue of the ability of new imaging techniques (MRI, PET/CT) in precisely localizing the lesion level, thus allowing spinal cord tumor excision with an acceptable morbidity rate [7]. The primary goal of surgery is decompression of the functional neural tissue followed by histological confirmation in case of isolated revealing tumor. Surgical resection is inappropriate in most patients, as some often suffer from other debilitating comorbidities. A recently published study reported prolonged median survival in selected patients who had undergone surgery and showed an improvement in their neurological status [5].

Radiotherapy has been employed either as a supplementary postoperative treatment or as a mainstay of treatment for ISCM, particularly for radiosensitive carcinomas. According to a current retrospective study, a 6-month survival rate was $36 \%$ with a median survival period of 4 months, when patients with ISCM received only radiotherapy at a total dose of 20-40 Gy. The majority of patients $(56 \%)$ had showed either neurological improvement or pain alleviation [8]. The risk of radiation myelitis is not negligible after a considerable amount of radiation exposure. Currently, much more targeted radiotherapy such as stereotactic radiotherapy is recommended. Anecdotal case reports have highlighted the role of fractionated stereotactic radiosurgery when other treatment options are unfeasible [9].

Overall prognosis of ISCM is generally sinister. Particularly, the mortality rate is $80 \%$ during the first three to four months after the manifestation of the first symptom $[1,2]$. The median survival rate is contingent on several factors such as the preoperative neurological status, nature of the primary cancer, and presence of systemic and/or central nervous system metastases [5, 7]. Furthermore, patients with breast cancer were found to have a favorable outcome compared to those with lung cancer or any other poorly differentiated primary tumors $[1,10]$.

In conclusion, ISCM is relatively uncommon and portends poor prognosis. Early recognition of 
this rare entity by means of MRI is of the utmost importance in promptly initiating therapeutic modalities, for preventing paraplegia and improving quality of life. Radiotherapy remains the main therapeutic approach albeit surgical resection should be considered as the primary treatment whenever feasible, particularly in the case of rapidly progressive neurological deficits and when a clear cleavage plane exists.

\section{References}

1. Kalayci M, Cağavi F, Gül S, Yenidünya S, Açikgöz B. Intramedullary spinal cord metastases: diagnosis and treatment-an illustrated review. Acta Neurochirurgica 2004; 146: 1347-1354.

2. Sung WS, Sung MJ, Chan JH, et al. Intramedullary spinal cord metastases: A 20-year institutional experience with a comprehensive literature review. World $J$ Neurosurg 2013; 79: 576-84.

3. Koutsis G, Spengos K, Potagas C, Dimitrakopoulos A, Sfagos K, Zakopoulos N. Intramedullary spinal cord metastases in a patient with small-cell lung cancer. Eur J Intern Med 2006; 17: 372-374.
4. Lee SS, Kim MK, Sym SJ, et al. Intramedullary spinal cord metastases: a single-institution experience. $\mathrm{J} \mathrm{Neu}$ rooncol 2007; 84: 85-89.

5. Dam-Hieu P, Seizeur R, Mineo JF, Metges JP, Meriot $\mathrm{P}$, Simon H. Retrospective study of 19 patients with intramedullary spinal cord metastasis. Clin Neurol Neurosurg 2009; 111: 10-17.

6. Metser U, Lerman H, Blank A, Lievshitz G, Bokstein F, Even-Sapir E. Malignant involvement of the spine: assessment by 18 F-FDG PET/CT. J Nucl Med 2004; 45: 279-284

7. Kalita O. Current insights into surgery for intramedullary spinal cord metastases: a literature review. Int J Surg Oncol 2011; 2011: 989506.

8. Hashii H, Mizumoto M, Kanemoto A, et al. Radiotherapy for patients with symptomatic intramedullary spinal cord metastases. J Radiat Res 2011; 52: 641-645.

9. Parikh S, Heron D. Fractionated radiosurgical management of intramedullary spinal cord metastases: A case report and review of the literature. Clin Neurol Neurosurg 2009; 111: 858-861.

10. Zebrowski A, Wilson L, Lim A, Stebbing J, Krell J. Intramedullary spinal cord metastases in breast cancer are associated with improved longer term systemic control. Future Oncol 2010; 6: 1517-1519.

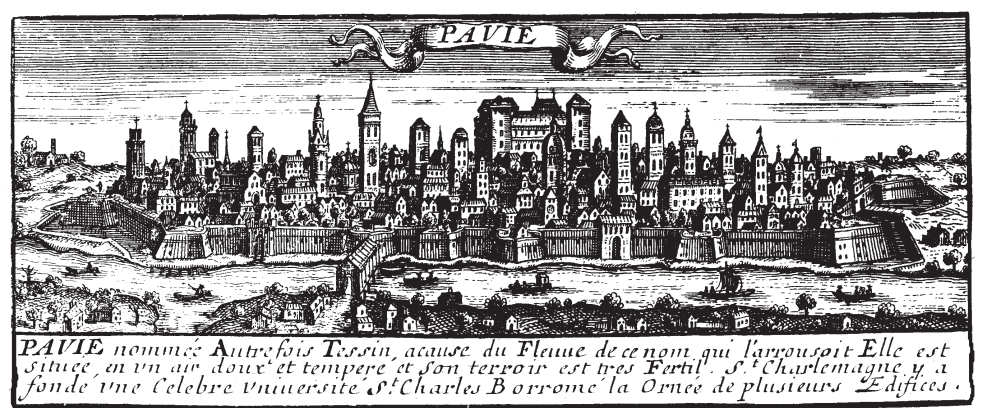

